\title{
Expectancies generated by recent exposure to melodic sequences
}

\author{
WILLIAM FORDE THOMPSON, LAURA-LEE BALKWILL, and ROXANA VERNESCU \\ York University, Toronto, Ontario, Canada
}

\begin{abstract}
In four experiments, we examined the effects of exposure to unfamiliar tone sequences on melodic expectancy and memory. In Experiment 1, 30 unfamiliar tone sequences (target sequences) were presented to listeners three times each in random order (exposure phase), and listeners recorded the number of notes in each sequence. Listeners were then presented target and novel sequences and rated how well the final note continued the pattern of notes that preceded it. Novel sequences were identical to target sequences, except for the final note. Ratings were significantly higher for target sequences than for novel sequences, illustrating the influence of exposure on melodic expectancy. Experiment 2 confirmed that without exposure to target sequences, ratings were equivalent for target and novel sequences. In Experiment 3, new listeners were assessed for explicit memory for target sequences following the exposure phase. Recognition of target sequences was above chance, but unrelated to expectancy judgments in Experiment 1. Experiment 4 replicated the exposure effect, using a modified experiment design, and confirmed that the effect is not dependent on explicit memory for sequences. We discuss the idea that melodic expectancies are influenced by implicit memory for recently heard melodic patterns.
\end{abstract}

This article concerns the influence of exposure to note sequences on melodic expectancy. To anticipate the results, exposure to melodic sequences influenced subsequent judgments of melodic expectancy. The influence could not be explained by conscious memory for sequences, supporting the view that melodic expectancies are generated by implicit memory for music. The findings are discussed in the light of research on melodic expectancy and implicit memory.

\section{Research on Melodic Expectancy}

Music listening involves the continuous formation of expectancies about subsequent events. The relationship between expected and actual events strongly affects our perception, memory, and aesthetic experience of music. A number of investigations have examined the role of expectancies in music listening. One line of research has addressed temporal expectancies, examining both the cues that give rise to such expectancies and the effects of conflicts between expected and actual events (e.g., Jones, 1990; Jones \& Boltz, 1989). Another line of research has addressed expectancies for pitch, focusing primarily on the cues that give rise to such expectancies (for a review, see Thompson \& Stainton, 1998).

This research was funded by a research grant from the Natural Sciences and Engineering Research Council of Canada to W.F.T. Doug Gifford provided technical assistance. We thank Robert Crowder, Lola Cuddy, Robert Greene, Peter Keller, and Elinor McKone for helpful comments on earlier drafts of the manuscript. Correspondence concerning this article should be addressed to W. F. Thompson, Department of Psychology, Atkinson College, York University, 4700 Keele Street, Toronto, Ontario M3J 1P3, Canada (e-mail: billt@yorku.ca).
Music theorists also have recognized the role of expectancies in music experience. Meyer (1956) argued that aesthetic responses to music are strongly affected by correspondences or conflicts between expected and actual events. Drawing from Meyer's ideas, Narmour (1990, 1992) proposed a formal model of melodic structure based on principles related to melodic expectancy. Narmour outlined three classes of influence on expectancies: innate or universal influences, called bottom-up implications; long-term knowledge of music, called extraopus style; and memory for recently heard patterns in the current melody, called intraopus style. As examples of the first two classes, pitch proximity (expecting notes that are proximal in pitch) is considered to be a universal influence on melodic expectancy, whereas diatonicism (expecting notes that are compatible with the scale) is thought to be based on long-term knowledge of Western music.

Empirical studies have addressed the first two classes of influence (e.g., Cuddy \& Lunney, 1995; Krumhansl, 1995a, 1995b; Schellenberg, 1996, 1997; Thompson, Cuddy, \& Plaus, 1997; Thompson \& Stainton, 1998). The aim of the present investigation was to illustrate the third class of influence on melodic expectancies and to evaluate whether this influence is an instance of implicit memory.

\section{Research on Implicit Memory}

It is widely acknowledged that memory is not always accompanied by conscious awareness, and there is a lengthy history of research and theory on unconscious memory. Ebbinghaus's (1885/1964) landmark studies on relearning exemplify the origin of contemporary approaches to the topic. His studies involved having partici- 
pants relearn a set of materials after a long retention interval and estimating the savings - the difference between the number of trials initially needed to learn the material and the number of trials needed to relearn that material at a later date. Later observations by Korsakoff (1889) provided further evidence for unconscious forms of memory. The behavior of amnesiacs often was influenced by recent experiences, even when those experiences were inaccessible to conscious memory.

Most research on implicit memory has involved visually presented stimuli, although auditory stimuli have also been examined (Schacter, 1987; Schacter, Chiu, \& Ochsner, 1993). In a typical experiment, participants are presented target stimuli in one phase and are later assessed for explicit (conscious) and implicit memory. Explicit memory may be assessed by using such tasks as free recall, cued recall, and recognition. Implicit memory may be assessed in various ways. In the stem completion task (e.g., Bassili, Smith, \& MacLeod, 1989; McKone \& Slee, 1997; Tulving, Schacter, \& Stark, 1982), participants are shown the first few letters of a word and are asked to produce an appropriate completion. In the fragment completion task (e.g., Greene, 1990; Roediger \& Blaxton, 1987), participants are shown a fragmented word and are asked to fill in the blanks. Implicit memory also has been demonstrated with preference ratings. Kunst-Wilson and Zajonc (1980) found that exposure to random dot patterns led to increased ratings of preference for those dot patterns, even when explicit memory for the patterns remained at chance levels (see also Moreland \& Zajonc, 1977, 1979).

The involvement of an implicit memory system is often inferred if the results of an implicit memory task are dissociable from explicit memory. Importantly, evidence for implicit memory is not restricted to circumstances in which explicit memory is absent. Participants may recall a test item when asked on an explicit test but may not employ explicit memory when performing an implicit test (Schacter, Bowers, \& Booker, 1989).

\section{Implicit Memory for Music}

Music experiences may be strongly affected by implicit memory. Crowder (1993) has argued that although some melodies are remembered explicitly, "the more ecologically general kinds of melody memory may occur implicitly ... the re-entry of a fugue theme, the occurrence of a lietmotif, or the developmental section of a remembered sonata-movement subject may all be examples of implicit music memory" (p. 134).

There are few studies of implicit memory for music. Johnson, Kim, and Risse (1985) reported evidence that amnesiac patients retain implicit memory for melodic sequences even when explicit memory is severely impaired. Alcoholic Korsakoff patients and nonalcoholic controls were played unfamiliar target melodies. Following a retention interval of $5 \mathrm{~min}$, participants rated their preference for both target and novel melodies. Both groups showed a similar increase in preference for melodies to which they had been exposed, a finding referred to as the exposure effect. However, the alcoholic group had significantly impaired explicit memory of the target melodies, in comparison with the control group.

Wilson (1979) observed a similar effect of exposure on preference for melodies. He employed a dichotic-listening procedure, in which melodic sequences were repeatedly exposed to an unattended ear while verbal material was simultaneously presented to an attended ear. In Experiment 1 , listeners were required to listen to the verbal material and proofread a text of that material. Subsequently, listeners rated test and novel sequences for overall preference and indicated whether each sequence was presented during the exposure phase. Preference ratings were higher for sequences that occurred in the exposure phase, and recognition also was above chance. In Experiment 2, another group of listeners performed the same task but, in addition, were required to shadow the verbal material (i.e., say each word aloud as they heard it). This additional task reduced the ability of listeners to process sequences in the unattended ear. This time, recognition was not significantly greater than chance, but preference ratings remained significantly higher for note sequences that occurred in the exposure phase (although the mean difference in preference ratings was considerably less than that found in Experiment 1). Wilson argued that the effect of exposure on preference judgments was independent of explicit memory for the sequences.

Peretz, Gaudreau, and Bonnel (1998) provided further evidence for this effect. They reported three experiments on the effect of exposure to melodies on preference and recognition. In each experiment, listeners were exposed to a set of familiar and unfamiliar melodies. Later, the exposed melodies, along with a set of matched distractor melodies, were presented. Half of the listeners in each experiment rated their preference for the test melodies (implicit task), and half identified those melodies they remembered hearing in the exposure phase (explicit task). Experiment 1 revealed that the effect of exposure on preference was limited to unfamiliar melodies and was evident both immediately after the exposure phase (test phase) and months later (retest phase). Recognition memory for exposed sequences was also above chance, however, which raises questions about the involvement of explicit memory in preference judgments. In Experiment 2, the researchers varied the time between the exposure and the test phases. Longer between-task intervals produced a greater decrease in preference for exposed melodies than did recognition for exposed melodies, illustrating a different temporal gradient of memory loss for affect and recognition. In Experiment 3, the timbre of sequences was made different in the exposure and the test phases. Recognition judgments were influenced by this change in timbre, whereas affect judgments were not. The authors concluded that recognition and affect are dissociable and that affect judgments are a form of implicit memory.

In addition to such effects on preference, exposure to music may have implicit effects for other kinds of judg- 
ments (Crowder, Serafine, \& Repp, 1990; Serafine, Crowder, \& Repp, 1984; Serafine, Davidson, Crowder, \& Repp, 1986; Thompson, Russo, \& Sinclair, 1994). The present study focused on melodic expectancy. ${ }^{1}$ There were two aims of the investigation. Our first aim was to evaluate Narmour's $(1990,1992)$ proposal that recent exposure to note sequences may influence melodic expectancy. Our second aim was to examine whether this influence reflects implicit memory for melody. To ensure that participants did not infer a connection between judgments of melodic expectancy and recognition of sequences, these variables were initially evaluated in different experiments. Judgments of melodic expectancy were obtained in Experiments 1 and 2, and recognition judgments were obtained in Experiment 3. Experiment 4 was designed to examine implicit and explicit memory within the same group of participants and to assess the possible involvement of negative priming.

\section{EXPERIMENT 1}

Experiment 1 was conducted to evaluate Narmour's proposal that melodic expectancies are influenced by recent exposure to melodic patterns. The experiment also extends studies by Wilson (1979) and Peretz et al. (1998), who found that recent exposure to melodic sequences affects subsequent judgments of preference. The present experiment differed in several important respects from these investigations. For example, Wilson employed a dichotic listening task in order to limit the depth of processing of melodic sequences: Verbal material was presented to the attended ear, and melodic sequences were presented to the unattended ear. In the present experiment, listeners were presented a series of brief note sequences to both ears and were required to record the number of notes in each. Because listeners attended to the number of notes, rather than to the patterns of pitch and duration, the depth of processing of sequences, and, hence, explicit memory for them, was reduced. Experiment 1 also involved a different rating task than that used by previous studies. Whereas the latter studies examined preference for melodic sequences, we asked listeners to judge the degree to which the final note of each sequence was expected.

\section{Method}

Participants. Twelve women and 8 men from the York University student population (mean age $=23$ years) volunteered for the experiment. All the participants reported normal hearing. The number of years of music training of the participants ranged from 0 to greater than 10, with an average of approximately 6 years of experience playing a musical instrument. The participants were naive with respect to the purpose of the experiment and were not familiar with the sequences.

Music sequences. Two sets (A and B) of 30 sequences, varying in length from four to nine notes, were composed for the experiment. The 30 sequences in Set $A$ are notated in Figure 1. Each sequence in Set A is identical to a sequence in Set B, except for the final note. The number of semitones between the last two notes of corresponding sequences in Sets A and B is identical, but the direction of pitch movement is opposite. For example, if the final two notes of a sequence in Set $A$ were $D_{4}-E_{4}$, the final two notes of the corresponding sequence in Set $B$ would be $D_{4}-C_{4}$. The number of sequences with descending and ascending endings is the same in the two sets. All the sequences are isochronous, with the duration of each note equal to $0.30 \mathrm{sec}$. Sequences were composed with a predominance of small melodic intervals: $42 \%$ of the intervals in sequences were two semitones or less, and $90 \%$ were seven semitones or less. Sequences were not composed in a tonal framework, but certain sequences may be interpreted as conforming, at least partially, to Western tonal conventions.

Apparatus. MIDI files for the 60 sequences were created using Encore software (Version 3.0.5). The tempo of all the sequences was 100 quarter-tone beats per minute, or $0.30 \mathrm{sec}$ per (eighth) note. The presentation of sequences was controlled by a Power Macintosh computer, using customized software (Experiment Creator). The computer was connected by a MIDI interface to a Roland Sound Canvas with equal temperament tuning. Sequence notes were the sampled piano sounds (piano 1) of the Roland Sound Canvas. The loudness for all the notes was set to the MIDI keypress velocity of 64. (Velocity values are scaled from 1 to 127.) The participants heard the sequences through Sennheiser HD-480 Classic headphones and were allowed to adjust the loudness to a comfortable listening level (approximately $70 \mathrm{~dB}$ SPL).

Procedure. The participants were tested individually. There were two phases: an exposure phase and a test phase. The participants took part in the exposure phase, which was followed by a pause of $10 \mathrm{~min}$, and then in the test phase. The participants were not informed of the test phase until after the exposure phase was completed, at which point they were told that there was a second experiment.

In the exposure phase, the 30 sequences in Set A were presented a total of three times. Sequences were presented in three blocks, and the order of presentation was randomized within blocks. The 90 sequences were each separated by a 0.90 -sec pause. The participants were required to count the number of notes in every sequence and to record each number on a form.

In the test phase, all 60 sequences (Set $\mathrm{A}$ and Set B) were presented in an order that was randomly and independently determined for each participant. The participants were required to rate the degree to which the final note of each sequence was expected. That is, they were required to rate whether the final note was a predictable continuation of the preceding notes, in a musical sense. Ratings were entered directly into the computer, using a scale from 1 to 7 , where a rating near 1 indicated that the final note was highly unexpected and a rating near 7 indicated that the final note was highly expected. The trials were self-paced, and the participants were debriefed on completion of the experiment.

\section{Results}

Ratings were subjected to an analysis of variance (ANOVA), with repeated measures on two factors: sequence (30 levels) and set ( 2 levels). As was predicted, expectancy ratings were higher for the set of sequences presented in the exposure phase (mean rating $=4.46$ ) than for the set of sequences not presented in the exposure phase [mean rating $=4.11 ; F(1,19)=6.50, p<.02$ ] This small but reliable difference supports the prediction that exposure to note sequences affects subsequent melodic expectancy. Moreover, the finding illustrates a highly specific effect of exposure, because matching sequences in the two sets were identical, except for the final (continuation) note. 


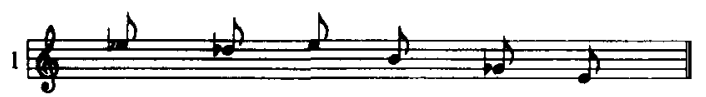

$11+2 \perp \perp \perp 2$

2201

$120+1$

$38 \div$

$13 \div$

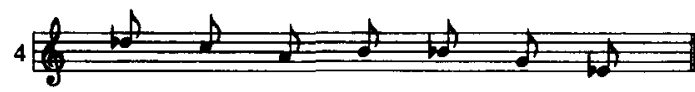

146

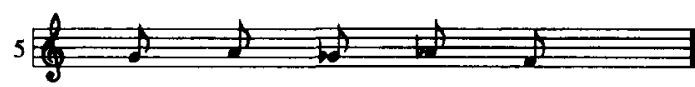

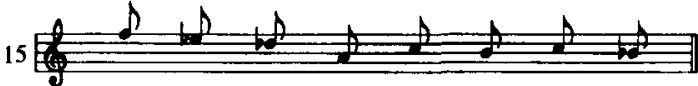

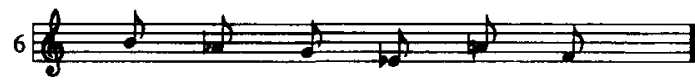

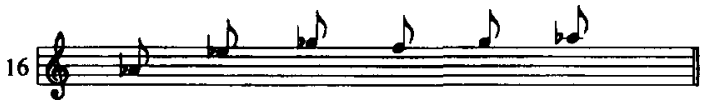

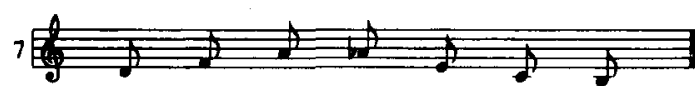

$172 D$
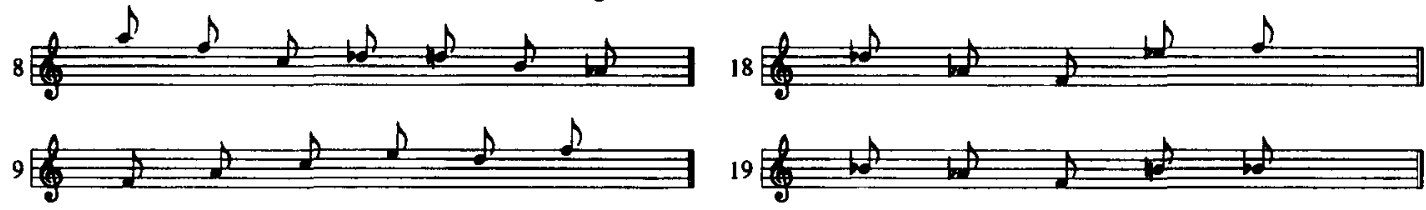

$19+212$
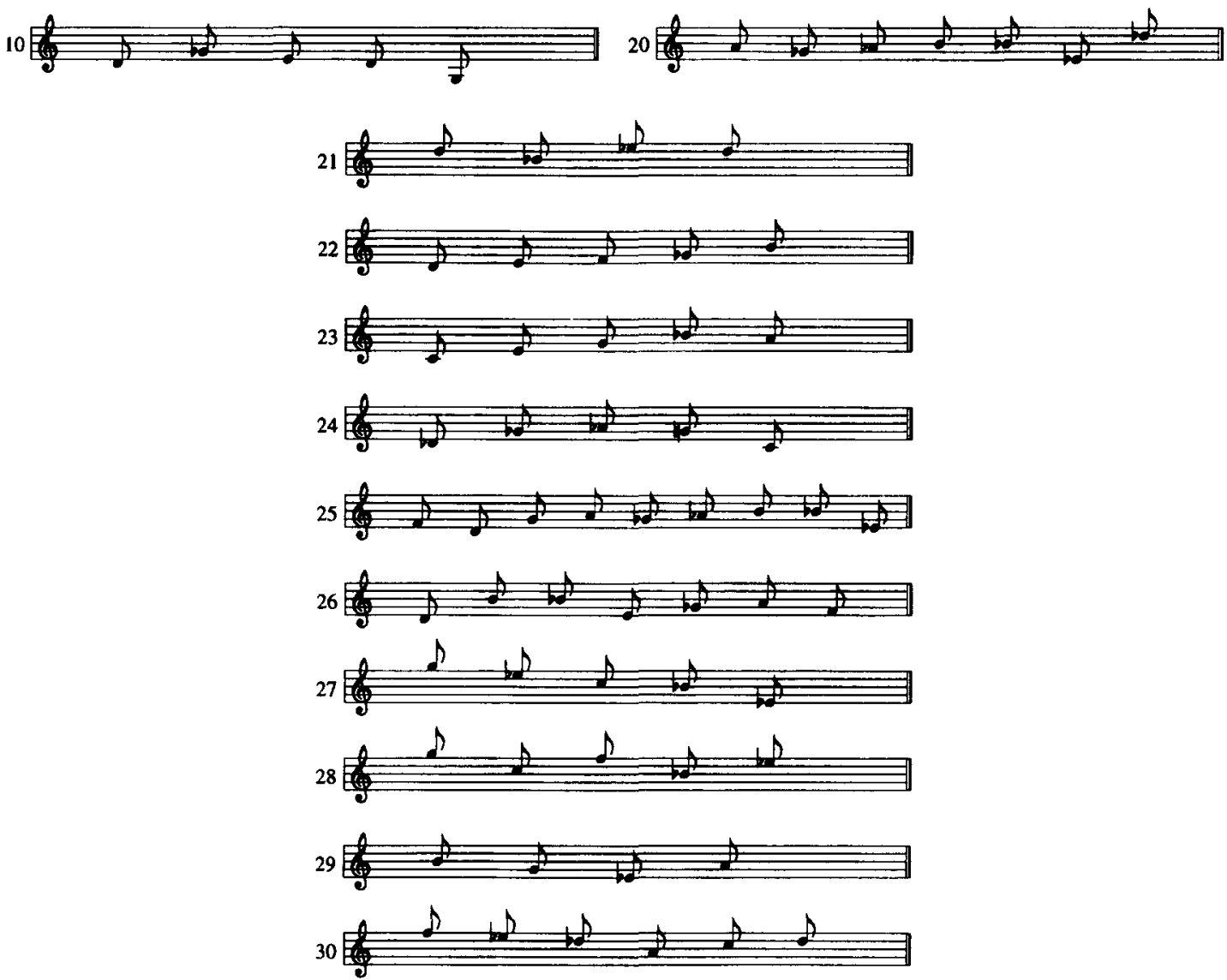

Figure 1. The 30 sequences (Set $A$ ) to which listeners were exposed. Matching sequences in Sets $A$ and $B$ are identical, except for the final note. The size of the final interval is identical in matching sequences, but the direction of pitch movement for that interval is opposite in the two sequences. 
The analysis also revealed a significant effect of sequence $[F(29,551)=3.26, p<.01]$ and a significant interaction between sequence and set $[F(29,551)=2.68$, $p<.01]$. The effect of sequence illustrates that regardless of exposure, expectancy ratings were higher for some sequences than for others. The interaction between sequence and set indicates that mean differences between sequences depended on the set of sequences considered.

To explore the latter effects, mean expectancy ratings for each of the 60 sequences were modeled as a function of various established predictors of expectancy (for a review, see Thompson \& Stainton, 1998), along with predictors based on the conditions of the present investigation. ${ }^{2} \mathrm{~A}$ four-predictor model accounted for a significant proportion of the variance in mean ratings $[R=.62$; $F(4,55)=8.41, p<.001]$. This model consisted of sequence length (i.e., number of notes; $b=-0.16, t=$ $-3.12, p<.01$ ), pitch proximity between the last two notes $(b=0.086, t=2.32, p<.05)$, diatonicism (i.e., whether notes could be drawn from a major scale; $b=$ $0.51, t=3.25, p<.01)$, and exposure $(b=0.30, t=2.15$, $p<.05)$. The predictive power of sequence length indicates that the participants assigned lower ratings as sequence length increased. Possibly, as sequence length increased, listeners were less able to assimilate that sequence into existing schemata, because most sequences were nontonal and unconventional (and hence unpredictable). The contribution of pitch proximity indicates that listeners generally expected the final note to be proximal in pitch to the penultimate note. The influence of pitch proximity is generally thought to be universal and has been demonstrated in numerous studies of melodic expectancy (for a review, see Thompson \& Stainton, 1998). The predictive power of diatonicism indicates that higher ratings were assigned if all the sequence notes were compatible with a major scale, suggesting that ratings were influenced by long-term knowledge of scale structure in Western music. Finally, the contribution of exposure corroborates the results of the ANOVA and indicates that exposure to sequences influenced ratings beyond what can be attributed to other predictors of expectancy.

\section{EXPERIMENT 2}

The results of Experiment 1 indicate that repeated exposure to note sequences influenced expectancy judgments. Experiment 2 was conducted to evaluate an alternative possibility. Although sequences in the two sets were matched except for the final note, it is possible that continuation notes in Set A (exposed set) would have been assigned higher ratings overall than those in Set B even without exposure. This possibility was considered remote, for three reasons. First, the two sets of sequences were identical, except for the final note of each matched pair. Second, the incidence of upward and downward melodic motion in the final two notes of sequences was balanced across the two sets. Third, aside from the latter constraint, the two sequences of each matched pair were assigned at random to Set A or Set B. Given such precautions, it would be highly improbable that, across 30 sequences in each set, a systematic bias in the predicted direction would emerge by chance. Nonetheless, to rule out this possibility, the participants in Experiment 2 provided expectancy ratings for all 60 sequences without prior exposure to either set.

\section{Method}

Participants. Eight women and 7 men from the University of New South Wales student population (mean age $=21$ years) volunteered for the experiment. All the participants reported normal hearing. The number of years of music training of participants ranged from 0 to greater than 10 , with an average of approximately 5 years of experience playing a musical instrument. The participants were naive with respect to the purpose of the experiment, and none had taken part in Experiment 1.

Sequences. The sequences were identical to those used in Experiment 1.

Apparatus. The apparatus was identical to that used in Experiment 1 .

Procedure. The participants took part in the test phase only. The procedure for the test phase was identical to that described for Experiment 1 .

\section{Results}

We did not expect a main effect of set, because the participants did not take part in an exposure phase and the two sets of sequences were identical, except for the final note. Expectancy ratings were indeed very similar for the sequences in Set $A($ mean $=4.49)$ and Set $B$ [mean $=4.50 ; F(1,14)<1.0$, n.s.]. This result supports the view that the difference in ratings observed in Experiment 1 resulted from exposure to sequences in Set $\mathrm{A}$, rather than from differences in the musical characteristics of the two sequence sets.

As in Experiment 1, there was a significant effect of sequence $[F(29,406)=2.62, p<.01]$ and a significant interaction between sequence and set $[F(29,406)=2.05$, $p<.01]$. These effects indicate that ratings of melodic expectancy were significantly higher for some sequences than for others and that these differences depend on the set of sequences considered. Multiple regression again revealed significant predictive power of pitch proximity $(b=0.14, t=3.92, p<.01)$ and diatonicism $(b=0.43$, $t=2.95, p<.01)$ and marginally significant predictive power of sequence length $(b=-0.09, t=-1.78, p<$ $.08)$. The multiple correlation for the regression model, $(.58)$ is highly significant $[F(3,56)=9.34, p<.01]$. These findings suggest that except for the influence of exposure, ratings of melodic expectancy were influenced by similar factors in Experiments 1 and 2, and there was a significant correlation between mean ratings of the 60 sequences in Experiments 1 and $2[r=.60 ; t(58)=5.64$, $p<.01]$.

\section{EXPERIMENT 3}

The results of Experiments 1 and 2 indicate that exposure to note sequences influenced judgments of melodic 
expectancy. Experiment 3 was conducted to assess explicit memory for sequences in the exposure phase. Our aim was to evaluate whether the influence of exposure on ratings of melodic expectancy could be attributed to explicit recognition of melodic sequences.

\section{Method}

Participants. Eleven women and 9 men from the York University student population (mean age $=24$ years) volunteered for the experiment. All the participants reported normal hearing. The number of years of music training of the participants ranged from 0 to greater than 10 , with an average of approximately 5 years of experience playing a musical instrument. The participants were naive with respect to the purpose of the experiment, and none had taken part in Experiment 1 or 2.

Sequences. The sequences were identical to those used in Experiment 1 .

Apparatus. The apparatus was identical to that used in Experiment 1 .

Procedure. The participants took part in the exposure phase, which was followed by a pause of $10 \mathrm{~min}$, and then in the test phase. The exposure phase was identical to that described for Experiment 1 . The participants were not informed of the test phase until after the exposure phase was completed.

In the test phase, all 60 sequences (Set $\mathrm{A}$ and Set B) were presented in random order. The participants were required to indicate whether they had heard each sequence during the counting task (exposure phase). Judgments were entered into the computer by selecting YES or NO on the computer screen. The participants were not given feedback on their judgments until the experiment was completed. The trials were self-paced.

\section{Results}

The participants reported recognizing an average of 13.10 of the 30 sequences presented in the exposure phase and 10.00 of the 30 novel sequences. This difference was reliable and indicates that the participants retained a small degree of explicit memory for sequences in the exposure phase $[t(19)=4.15, p<.02]$.

Unlike ratings of melodic expectancy, the probability of making a recognition response was unrelated to sequence length $(r=.13, d f=58$, n.s. $)$, pitch proximity $(r=$ .10$, n.s. $)$, or diatonicism $(r=.11, d f=58$, n.s.). (Multiple regression analyses also failed to reveal a relationship with any of these variables.)

We next examined whether exposure to sequences affected recognition and expectancy in similar ways. Mean ratings of the 60 sequences in Experiment 1 were regressed on three predictors: exposure to sequences (dummy coded), the probability of making a recognition response (Experiment 3), and mean ratings when there was no exposure phase (Experiment 2). If the exposure effect observed in Experiment 1 were based merely on recognition of sequences, exposure to sequences should contribute no predictive power beyond that provided by recognition. Conversely, if judgments of melodic expectancy reflected implicit memory for previously heard sequences, exposure to sequences should have predictive power beyond that provided by recognition.

There were two significant predictors: mean ratings when there was no exposure phase $[b=0.66, t(57)=$
$6.00, p<.01]$ and exposure to sequences $[b=0.35$, $t(57)=2.68, p<.01]$. The multiple correlation $(.65)$ was highly significant $[F(2,57)=21.20, p<.01]$. Recognition of sequences provided no predictive power beyond that provided by these predictors (partial correlation $=$ .04, n.s.).

The correlation between mean ratings of expectancy (Experiment 1) and recognition (Experiment 3) (.08) was insignificant $[t(58)<1.0$, n.s. $]$. In contrast, the correlation between mean ratings of expectancy in Experiments 1 and $2(.60)$ was highly significant $[t(58)=5.64, p<$ $.001]$. The latter finding is important because it suggests that the insignificant correlation between expectancy ratings (Experiment 1) and recognition responses (Experiment 3) cannot be explained merely in terms of low reliability in Experiment 1 or between-group error variance. In short, exposure to sequences appears to have influenced ratings of melodic expectancy beyond what can be explained by recognition of sequences. Nonetheless, because recognition and expectancy judgments were obtained from different groups of participants, it is still possible that the participants in Experiment 1 were influenced by explicit memory for sequences. Experiment 4 was conducted to examine the issue further.

\section{EXPERIMENT 4}

The results of Experiments 1, 2, and 3 suggest that exposure to note sequences influenced judgments of melodic expectancy and that these judgments cannot be attributed to explicit recognition of those melodic sequences. Experiment 4 addressed three remaining issues. First, the effect of exposure on expectancy judgments, although reliable, was small. Thus, the first aim of Experiment 4 was to replicate the exposure effect, using a modified design. Second, the possibility that explicit memory influenced expectancy judgments in Experiment 1 cannot be entirely ruled out, because some explicit memory for sequences was observed in Experiment 3. Experiment 4 addressed this issue by directly asking listeners whether any of their expectancy ratings were influenced by memory for sequences heard in the exposure phase. We also reduced the likelihood of any explicit memory for sequences in Experiment 4 by reducing the depth of processing of sequences during the exposure phase. Specifically, the participants were required to classify rapidly a series of words as nouns or verbs (see the Method section).

Third, a comparison of mean ratings in Experiments 1 and 2 raises the possibility that negative priming was involved in the exposure effect. Experiment 1 revealed that ratings of exposed sequences (Set A) were significantly higher than ratings of unexposed sequences (Set B), confirming that some form of priming had occurred. Surprisingly, however, ratings of exposed sequences in Experiment 1 (Set A) were no higher than ratings of those same sequences in Experiment 2 (in which there was no exposure phase). Instead, ratings of the unexposed se- 
quences in Experiment 1 (Set B) were lower than ratings of those same sequences in Experiment 2. We interpreted these observations as simple criterion differences between the participants in Experiments 1 and 2 (which were sampled from universities in Canada and Australia, respectively), but they may also be interpreted as an instance of negative priming. That is, the effect of exposure may have been to decrease ratings for unexposed-related sequences, rather than to increase ratings for Exposed sequences. Experiment 4 was designed to clarify whether the effects of exposure on expectancy judgments are best characterized as positive or negative priming.

\section{Method}

Participants. Four groups of 10 students (27 women and 14 men) at York University participated in the experiment and received course credit for their participation. The mean age was approximately 26 years. The majority of them played at least one musical instrument $(85 \%)$, with an average of 5 years of formal training. Few of them were still actively performing $(30 \%)$, with an average of 10 years having elapsed since they last played. The participants were naive with respect to the purpose of the experiment, and none had participated in Experiments 1, 2, or 3.

Sequences. The sequences were identical to those used in Experiment 1. However, the 30 sequences in Set A were classified into two sets of 15 sequences, labeled Sets 1 and 2 , and the 30 sequences in Set B were classified into two sets of 15 sequences, labeled Sets 3 and 4.

Apparatus. The apparatus was identical to that used in Experiment 1 .

Procedure. The participants were exposed to four repetitions of 15 different sequences, followed by a pause of $10 \mathrm{~min}$ and then by the test phase. The 15 sequences within each repetition were presented in a random order. Throughout the exposure phase, the participants performed a distraction task. Written sentences were displayed on the computer screen, with one word underlined (e.g., Mary wants to drink her juice). The participants were required to rapidly classify the underlined word as a noun or a verb by making a selection with the computer mouse. If no response was made within $1 \mathrm{sec}$, the trial was recorded as incorrect, and the next sentence appeared on the computer screen. The percentage of correct responses was displayed on the screen and updated after each response. The participants were told that their performance on this task would be recorded and that they should attempt to achieve as close as possible to $100 \%$ accuracy.

During the exposure phase, each of the four groups was exposed to a different set of 15 sequences (Set 1,2,3, or 4). Sets 1 and 2 were the 30 sequences referred to as Set $A$ in Experiments 1-3; Sets 3 and 4 were the 30 sequences referred to as Set $B$ in Experiments $1-3$. Recall that the sequences in Set $A$ (Sets 1 and 2) were identical to the sequences in Set B (Sets 3 and 4), except for the final note of each matched pair. For the participants in Group 1, who were exposed to Set 1 , Set 1 was labeled exposed; Set 2 was labeled unexposed-unrelated; Set 3 was labeled unexposed-related; and Set 4 was labeled unexposed-unrelated.

The participants were not informed of the test phase until after they had completed the exposure phase. In the test phase, all $60 \mathrm{se}$ quences were presented in a random order. The participants were required to rate the degree to which the final note of each sequence was expected, on a scale from 1 (highly unexpected) to 7 (highly expected). The trials were self-paced.

After completing the test phase, the participants were asked to describe any factors that influenced their expectancy judgments. They were then asked whether their ratings were influenced by recognition of, or familiarity with, the test sequences.

\section{Results}

The four sets of ratings for all the groups were labeled as follows: Set 1, exposed; Set 2, unexposed-unrelated; Set 3, unexposed-related; and Set 4, unexposedunrelated. Note that across the four groups of participants, each of the 60 sequences occurred an equal number of times in each set. The exposed sequences (Set 1) consisted of the 15 sequences presented to the participants during the exposure phase (which differed for each group). The unexposed-related sequences (Set 3) were always identical to the exposed sequences, except for the final note. The two sets of unexposed-unrelated sequences consisted of 15 sequences that were unrelated to the exposed sequences but related to each other (i.e., identical except for the final note).

Mean ratings for the four sets were assessed in a repeated measures ANOVA. The analysis revealed a main effect of set $[F(3,117)=5.64, p<.005]$, with significantly higher ratings assigned to exposed sequences (Set 1 , mean $=4.50$ ) than to unexposed sequences [Sets 2,3 , and 4 , mean rating $=4.27 ; F(1,117)=9.72, p<$ $.005]$. This finding replicates the effect of exposure on melodic expectancy observed in Experiment 1. Ratings were also significantly higher for exposed sequences than for unexposed-related sequences [Set 3 , mean $=4.21$; $F(1,117)=9.92, p<.005]$. This finding indicates that the priming effect is highly specific, in that these two sets of sequences were identical except for the final note of each matched pair.

The latter result can be explained as either positive priming (increased ratings for exposed sequences) or negative priming (decreased ratings for unexposedrelated sequences). Two additional comparisons, however, suggested that only positive priming was involved. First, ratings were significantly higher for exposed sequences (Set 1 , mean $=4.50$ ) than for unexposed-unrelated sequences [Sets 2 and 4 , mean $=4.29 ; F(1,117)=6.71, p<$ $.02]$, confirming the involvement of positive priming. Second, ratings were not significantly different for unexposed-related sequences (Set 3, mean $=4.21$ ) than for unexposed-unrelated sequences [Sets 2 and 4, mean rating $=4.29 ; F(1,117)=1.10, p=0.30]$, as would be expected if negative priming were involved. Taken together, the results support positive priming, but not negative priming.

When asked to describe any factors that influenced their expectancy judgments, none of the participants cited memory of the test sequences. For this question, the participants provided only very general responses, such as, "if the last note made sense in the context of the other notes, I gave a high rating." When asked specifically whether their ratings were influenced by recognition of the test sequences, all 40 participants responded "no."

\section{GENERAL DISCUSSION}

The results of this investigation illustrate that exposure to unfamiliar note sequences influenced judgments 
of melodic expectancy. Listeners assigned higher ratings of expectancy to sequences presented in the exposure phase than to novel sequences. To our knowledge, these results provide the first empirical support for Narmour's $(1990,1992)$ proposal that melodic expectancies are influenced by recent exposure to note sequences. The findings also extend results by Wilson (1979) and Peretz et al. (1998), by illustrating that exposure to note sequences not only affects preference judgments, but also affects melodic expectancy. The effect of exposure was small, but this was to be expected: Melodic expectancies are shaped and reinforced from an early age, and we did not expect exposure to novel sequences to overwhelm such long-term influences on expectancy.

Experiment 3 revealed a small degree of recognition of sequences presented in the exposure phase. This finding was not anticipated, because the exposure phase was designed to suppress explicit recognition of sequences. Attending to the number of notes in each sequence should have prevented the participants from abstracting musical features useful for recognition, such as scale structure, melodic contour, interval pattern, and hierarchical organization (see, e.g., Cohen, 1991; Deutsch \& Feroe, 1981; Dowling \& Harwood, 1986). Presumably, the participants either performed the counting task without devoting all of their attention to sequence length or encoded other features of sequences unconsciously and automatically. Nonetheless, further analyses suggested that the effects of exposure on recognition were unrelated to the effects of exposure on expectancy.

The role of explicit memory was reexamined in Experiment 4. In this case, the participants performed a verbal classification task during the exposure phase, further reducing the depth of processing of melodic sequences. Once again, exposure to sequences significantly influenced expectancy judgments, and the effect appeared to be implicit: When asked to specify all of the factors that influenced their expectancy judgments, no participants reported any influence by explicit memory for sequences. In Experiment 4, the possible involvement of negative priming in the exposure effect was also examined, but no evidence for negative priming was observed.

The effect of exposure to novel melodies on expectancy has important implications for music experience. According to Meyer (1956), the relationship between expected and actual notes powerfully shapes our aesthetic responses to music. When expectations are violated, listeners experience an arousal response, leading to a reevaluation of the stimulus and the experience of emotion. The emotion experienced is thought to depend on both the level of arousal and the properties of the musical stimulus.

Narmour $(1990,1992)$ extended this discussion by arguing that the relationship between expected and actual events also determines the perception of melodic structure. Narmour proposed three classes of influence on expectancy, two of which have been empirically assessed: (1) innate sensitivity to melodic implications, described as bottom-up principles, and (2) long-term knowledge of music, or extraopus style (Cuddy \& Lunney, 1995; Krumhansl, 1995a, 1995b; Schellenberg, 1996, 1997; Thompson et al., 1997; Thompson \& Stainton, 1998). The present findings illustrate the third class of influence identified by Narmour: memory for recently heard melodic patterns, or intraopus style. The study also extends Narmour's analysis of intraopus style by suggesting that this influence may be implicit.

Finally, the results provide insight as to how listeners might develop an appreciation for nontonal or unconventional music. Melodic expectancies were affected by limited exposure to novel sequences composed in a nontonal, unfamiliar style. With repeated exposure to melodies composed in an unfamiliar but consistent style, listeners should eventually develop stable expectancies for such melodic patterns. As these expectancies become widespread among listeners, composers may then create patterns of denials and fulfillments of these established expectancies, shaping the listeners' aesthetic responses as the music unfolds.

\section{REFERENCES}

Bassili, J. N., Smith, M. C., \& MacLeod, C. M. (1989). Auditory and visual word stem completion: Separating data-driven and conceptuallydriven processes. Quarterly Journal of Experimental Psychology, 41A, 439-453.

BerLyne, D. E. (1970). Novelty, complexity, and hedonic value. Perception \& Psychophysics, 8, 279-286.

CoHEN, A. J. (1991). Tonality and perception: Musical scales primed by excerpts from The Well-Tempered Clavier of J. S. Bach. Psychological Research, 53, 305-314.

Crowder, R. G. (1993). Auditory memory. In S. McAdams \& E. Bigand (Eds.), Thinking in sound: The cognitive psychology of human audition (pp. 113-145). Oxford: Oxford University Press, Clarendon Press.

Crowder, R. G., Serafine, M. L., \& Repp, B. (1990). Physical interaction and association by contiguity in memory for the words and melodies of songs. Memory \& Cognition, 18, 469-476.

Cuddy, L. L., \& Lunney, C. A. (1995). Expectancies generated by melodic intervals: Perceptual judgments of melodic continuity. Perception \& Psychophysics, 57, 451-462.

DeUTSCH, D., \& FEROE, J. (1981). The internal representation of pitch sequences in tonal music. Psychological Review, 88, 503-522.

Dowling, W. J., \& HARWood, D. L. (1986). Music cognition. Orlando, FL: Academic Press.

EbBinghaus, H. (1964). Memory: A contribution to experimental psychology (H. A. Ruger \& C. E. Bussenius, Trans.). New York: Dover. (Original work published 1885)

GREENE, R. (1990). Spacing effects on implicit memory tests. Journal of Experimental Psychology: Learning, Memory, \& Cognition, 16, 1004-1011.

HEYDUK, R. G. (1975). Rated preference for musical compositions as it relates to complexity and exposure frequency. Perception \& Psychophysics, 17, 84-90.

Johnson, M. K., KIM, J. K., \& Risse, G. (1985). Do alcoholic Korsakoff's syndrome patients acquire affective reactions? Journal of $E x$ perimental Psychology: Learning, Memory, \& Cognition, 11, 22-36.

JoNES, M. R. (1990). Learning and the development of expectancies: An interactionist approach. Psychomusicology, 9, 193-228.

Jones, M. R., \& BolTZ, M. (1989). Dynamic attention and responses to time. Psychological Review, 96, 459-491.

KORSAKOFF, S. S. (1889). Étude medica-psychologique sur une forme des maladies de la memoire [Medical-psychological study of a form of diseases of memory]. Revue Philosophique, 28, 501-530. 
Krumhansl, C. L. (1995a). Music psychology and music theory: Problems and prospects. Music Theory Spectrum, 17, 53-80.

KRUMHANSL, C. L. (1995b). Tonal and melodic implications of musical intervals. Systematische Musikwissenschaft, 3, 211-250.

KUNST-WILSON, W. R., \& ZAJONC, R. B. (1980). Affective discrimination of stimuli that cannot be recognized. Science, 207, 557-558.

MCKONE, E., \& SLEE, J. A. (1997). Explicit contamination in "implicit" memory for new associations. Memory \& Cognition, 25, 352-366.

MEYER, L. B. (1956). Emotion and meaning in music. Chicago: University of Chicago Press.

Moreland, R. L., \& Zajonc, R. B. (1977). Is stimulus recognition a necessary condition for the occurrence of exposure effects? Journal of Personality \& Social Psychology, 35, 191-199.

Moreland, R. L., \& ZaJonC, R. B. (1979). Exposure effects may not depend on stimulus recognition. Journal of Personality \& Social Psychology, 37, 1085-1089.

NaRMOUR, E. (1990). The analysis and cognition of basic melodic structures. Chicago: University of Chicago Press.

NARMOUR, E. (1992). The analysis and cognition of melodic complexity. Chicago: University of Chicago Press.

Peretz, I., Gaudreau, D., \& Bonnel, A.-M. (1998). Exposure effects on music preference and recognition. Memory \& Cognition, 26, 884902.

Roediger, H. L., III, \& Blaxton, T. A. (1987). Effects of varying modality, surface features, and retention interval on priming in wordfragment completion. Memory \& Cognition, 15, 379-388.

SCHACTER, D. L. (1987). Implicit memory: History and current status. Journal of Experimental Psychology: Learning, Memory, \& Cognition, 13, 501-518.

SCHACTER, D. L., BOWERs, J., \& BOOKER, J. (1989). Intention, awareness, and implicit memory: The retrieval intentionality criterion. In S. Lewandowsky, J. Dunn, \& K. Kirsner (Eds.), Implicit memory: Theoretical issues (pp. 47-65). Hillsdale, NJ: Erlbaum.

SCHACTER, D. L., ChIU, P., \& OChSNER, K. H. (1993). Implicit memory: A selective review. Annual Review of Neuroscience, 16, 159-182.

SCHEllenberG, E. G. (1996). Expectancy in melody: Test of the implication-realization model. Cognition, 58, 75-125.

SCHELlenBerg, E. G. (1997). Simplifying the implication-realization model. Music Perception, 14, 295-318.

Serafine, M. L., Crowder, R. G., \& REPP, B. H. (1984). Integration of melody and text in memory for songs. Cognition, 16, 285-303.

Serafine, M. L., Davidson, J., Crowder, R. G., \& Repp, B. H. (1986). On the nature of melody-text integration in memory for songs. Journal of Memory \& Language, 25, 123-135.

THOMPSON, W. F. (1996). Eugene Narmour: The analysis and cognition of basic melodic structures (1990) and The analysis and cognition of melodic complexity (1992): A review and empirical assessment. Journal of the American Musicological Society, 49, 127-145.

Thompson, W. F., Cuddy, L. L., \& Plaus, C. (1997). Expectancies generated by melodic intervals: Evaluation of principles of melodic im- plication in a melody-completion task. Perception \& Psychophysics, 59, 1069-1076.

THOMPSON, W. F., Russo, F. A., \& SinClair, D. (1994). Effects of underscoring on the perception of closure in film excerpts. Psychomusicology, 13, 9-27.

Thompson, W. F., \& Stainton. M. (1998). Expectancy in Bohemian folksong melodies: Evaluation of implicative principles for implicative and closural intervals. Music Perception, 15, 231-252.

Tulving, E., Schacter, D. L., \& Stark, H. A. (1982). Priming effects in word-fragment completion are independent of recognition memory. In P. E. Morris \& M. A. Conway (Eds.), The psychology of memory III (pp. 333-339). New York: New York University Press.

WILSON, W. R. (1979). Feeling more than we can know: Exposure effects without learning. Journal of Personality \& Social Psychology, 37, 811-821.

\section{NOTES}

1. The relationship between expectancy and preference has not been established, but it is clear that what we expect is not always what we prefer. Meyer (1956) proposed that when expectancies are violated, an arousal response may occur. This arousal is thought to be a precursor to emotion, with the precise emotion experienced being dependent on other stimulus characteristics. Another theory suggests that expectancy and preference are related by a nonlinear (unimodel) function (Berlyne, 1970; Heyduk, 1975). According to Berlyne's optimal complexity model, listeners prefer intermediate levels of complexity. Complexity is a psychological attribute that varies with predictability. Thus, higher levels of preference may be associated with intermediate levels of predictability, whereas lower levels of preference may be associated with either high or low levels of predictability.

2 . We assessed the following additional predictors based on Narmour's (1990) model of melody: intervallic difference, registral direction, registral return, and closure. Intervallic difference predicts that listeners expect a small interval (less than six semitones) to be followed by a similar-sized interval, and a large interval to be followed by a relatively smaller interval. Registral direction predicts that listeners expect a small interval to be followed by movement in the same registral direction, but a large interval to be followed by a change in registral direction. Registral return predicts that after any interval, listeners expect a pitch near or at the first note of an interval. Closure predicts that after any interval, listeners expect a change in direction, movement to a smaller interval, or both. For reviews of Narmour's model, see Krumhansl (1995a) or Thompson (1996). Because we did not systematically vary the size of the penultimate interval and assess a wide range of continuation notes, this investigation is not ideally suited to a formal assessment of Narmour's principles.

(Manuscript received April 21, 1997; revision accepted for publication July 28 , 1999.) 